\title{
Transactive control: a framework for operating power systems characterized by high penetration of distributed energy resources
}

\author{
${\text { Junjie } \mathrm{HU}^{1}{ }^{1} \text {, Guangya YANG }}^{1}$, Koen KOK ${ }^{1,2}$, Yusheng XUE ${ }^{3}$, \\ Henrik W. BINDNER ${ }^{1}$

\author{
(1)
} \\ Y
}

(n)

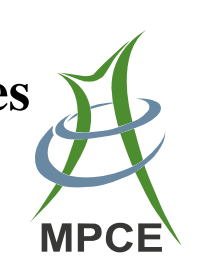

\begin{abstract}
The increasing number of distributed energy resources connected to power systems raises operational challenges for the network operator, such as introducing grid congestion and voltage deviations in the distribution network level, as well as increasing balancing needs at the whole system level. Control and coordination of a large number of distributed energy assets requires innovative approaches. Transactive control has received much attention due to its decentralized decision-making and transparent characteristics. This paper introduces the concept and main features of transactive control, followed by a literature review and demonstration projects that apply to transactive control. Cases are then presented to illustrate the transactive control framework. At the end, discussions
\end{abstract}

CrossCheck date: 16 May 2016

Received: 13 January 2016/Accepted: 16 May 2016/Published online: 23 August 2016

(C) The Author(s) 2016. This article is published with open access at Springerlink.com

$凶$ Junjie HU

junhu@elektro.dtu.dk

Guangya YANG

gyy@elektro.dtu.dk

Koen KOK

koenkok@elektro.dtu.dk

Yusheng XUE

xueyusheng@sgepri.sgcc.com.cn

Henrik W. BINDNER

hwbi@elektro.dtu.dk

1 Center for Electric Power and Energy, Technical University of Denmark, Copenhagen, Denmark

2 Netherlands Organization for Applied Research, TNO, The Hague, The Netherlands

3 State Grid Electric Power Research Institute, Nanjing, China and research directions are presented, for applying transactive control to operating power systems, characterized by a high penetration of distributed energy resources.

Keywords Distributed energy resources, Distribution system operation, Smart grids, Transactive control

\section{Introduction}

An important means used by the power industry to reduce greenhouse-gas emissions and fossil-fuel dependency is the introduction of renewable energy generation such as wind and solar [1-3]. As part of this transition to sustainability, the majority of new generation units are being connected to distribution grids $[4,5]$. In addition, new loads are being introduced, such as electric vehicles and heat pumps. Although these new loads lead to higher overall energy efficiency, their introduction leads to higher electricity use and thus, to higher power loading of the power grid. These new energy resources bring challenges to power system operation, including a decline in reserve power from traditional sources [6], and grid congestion [7]. In this paper, electric vehicles, heat pumps etc. flexible loads, storages, and distributed generation, including renewable generation units such as wind turbine and photovoltaic generation, are generally referred to distributed energy resources (DER). In order to cope with the challenges described above, DER needs to be become actively involved in grid coordination and operation tasks; and the demand response scheme of the DER needs to be introduced to make available additional operational flexibility [8].

The problems encountered in the power systems have received much attention, and various efforts have been made to address the problems. These range from 
developing new control methods for individual component operation [9] to radical rethinking of system operations [10-13]. In [11-13], the need for coordination between transmission system operator (TSO) and distribution system operator (DSO) operation are discussed. Traditionally, the system is kept secure by centralized control actions. For example, the transmission system operator centrally controls few big power plants through a supervisory control and data acquisition system. The distribution system operator centrally manages the status of key devices, such as breakers, reference setting points of on/off load tap changers, capacity banks, etc. However, it is impossible to control a large number of distributed energy resources today [8], as the grid control systems are centralized by design, and do not yet actively integrate distributed energy resources into the operation on a meaningful scale.

The current development of smart grid technologies allows DERs to adjust their operation through on-site local monitoring and computing, and receive remote-control signals from a market or grid operator using information communication technology (ICT). It is likely that the electrical power system will evolve into a hybrid system in the next 20 years, with a set of primary generation, storage, and transmission systems coordinated through the market, and a set of distributed resources managed on a decentralized basis, using a mechanism that can leverage the capabilities of DER systems [8]. The control and coordination of a large number of distributed energy resources requires innovative distributed approaches. Transactive control is such an approach.

Transactive control is a form of market-based control that has been adopted by several projects and initiatives $[14,15]$. The intent of transactive control is to reach equilibriums by standardizing a scalable, distributed mechanism via exchanging information about generation, consumption, constraints, and responsive assets over dynamic, real-time forecasting periods, using economic incentive signaling [16], and thus solving complex power system problems. In another words, the operation is based on the management of interactions instead of actions.

This paper reviews the recent development in transactive control application, and illustrates that as a framework which can be applied generally to the operation of power systems characterized by a high penetration of distributed energy resources. By reviewing the existing demonstration projects and literature, and presenting illustrative case studies, the paper classifies the implementing methods of transactive control by way of information exchange between the involved actors and the operational purpose. Furthermore, the research challenges faced by the application of transactive control in future power systems are discussed.
The remainder of the paper is organized as follows: concepts and main methods of transactive control are introduced in Section 2. Section 3 reviews the literature that applies to the transactive control framework to manage distributed energy resources. In Section 4, demonstration projects are described. Section 5 presents two case studies using the transactive control framework. Research challenges are discussed in Section 6. Finally, a discussion and conclusion are given in Section 7.

\section{Transactive control in smart grids}

\subsection{Basic concept}

A transaction is an exchange of goods, services and/or funds through negotiation. Accordingly, transactive control is a framework that enables actors to interact with each other through an economic signal, in order to optimize the allocation of resources. In [14], transactive control is defined as an implementation of transactive energy. Transactive Energy is "a set of economic and control mechanisms that allows the dynamic balance of supply and demand across the entire electrical infrastructure using value as a key operational parameter" [14]. In a transactive energy-management system, mid- to small-sized electricity-consuming or producing devices automatically negotiate their actions with each other, with devices in the physical network, and with dispatch systems of energy suppliers through efficient and scalable electronic market algorithms [17].

In fact, transactive control is already in use in some wholesale energy markets in the world, for instance the Nord Pool Spot market [18]. The trading and clearing mechanism used in the market is a typical form of transactive control. However, the application of transactive control is still largely missing in distribution system operation and at the retail market level.

In the transactive control framework, the utility function applied in microeconomics is used, and describes the degree of well-being the product provides for consumers. It thus defines the different responses of different devices to various prices [19]. As a framework, its application in power systems can be classified into the following aspects, which will be discussed further in Sections 3 and 4.

1) Frequency regulation via tertiary control [20].

2) Frequency control in power systems via secondary control [19].

3) Congestion and voltage management in distribution network [7, 21-23].

4) Manage the energy distribution inside an operation of balance responsible parties (or aggregators) [24, 25]. 
5) Distribution system operation in term of influencing the behaviors of the decision-makers connected on the network [26].

6) New electricity spot market mechanism that facilitates the participation of flexible demand [27, 28].

7) Residential optimal energy management considering network operational constraints of utility [29-31].

\subsection{Time scale}

In transactive control, one of the main ideas is to integrate retail and wholesale markets, and other markets, into a single platform, by utilizing forward and spot transactions, thereby guiding investment and operating decisions [32]. Forward transaction is used to coordinate investment decisions and manage risks. Spot transaction is used to coordinate operating decisions and mitigate risk. The time scale of these two type transactions is shown in Fig. 1. Forward transactions are not new compared to the current market mechanism. However, the spot could be real time, or close to real time, e.g., 5 minutes. In the demonstration projects described in Section 4, a 5-minute market is widely used.

\subsection{Implementing methods}

In general, two types of implementing methods are widely used in the literature, and in the demonstration projects that will be introduced in Sections 3 and 4. The implementing method is defined as a way to find the equilibrium among the actors, and thus complete the transactions. These two kinds of approaches include:

1) A one-time information exchange-based method, such as the merit-order-based market-clearing mechanism.

2) An iterative information exchange-based method that, mathematically, is normally explained by dual decomposition computing algorithms.

In the demonstration projects, the one-time information exchange-based method is widely applied. Each

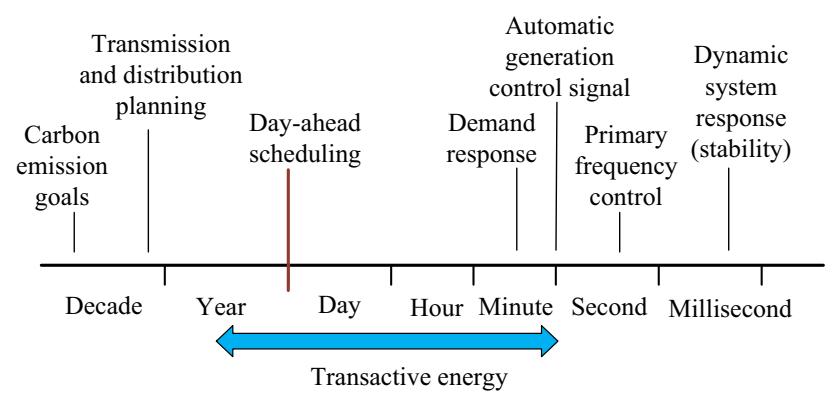

Fig. 1 Transactive control's position in electric power system timelines when applying for system operation participator generates a bid (a quantity and a price) for every bid period (e.g., 5 minutes), and communicates with the aggregating entity. Figure 2 shows an example of the method to solve a grid congestion problem. Three steps are included in the transactive control. Firstly, each device (or device agent) bids their available flexibility to the aggregating entity (system operator in this case). Then, the system operator aggregates the bids and performs a pricediscovery mechanism; i.e., a merit-order-based marketclearing mechanism to find the clearing price. The cleared price is used to control the devices. We note that meritorder-based market-clearing mechanism is widely used to find the price, and to realize the transactions in the demonstration projects.

Besides the one-time information exchange, an iterative information exchange-based method is also widely proposed that normally exploits the algorithms of dual decomposition $[34,35]$. Note that in transactive control framework, the dual variables are normally interpreted as prices that reflect the equilibriums, while in many studies they only serve as the coordinating signals [34, 35]. Figure 3 illustrates the scheme of the method. Examples are given in the figure where the interaction between system operator/aggregators and aggregators/DER units are presented. Note that the upper-level entity sends the price to the lower-level entity; in correspondence, the lower level responds with its power schedules. After certain iterations, an agreement is reached between the upper-level entity and the lower-level entity. In our view, the upper-level entity decides when to stop based on its operational purpose.

Note that the key difference between these two approaches is the information exchange timing. The onetime information exchange-based method is less complex, leading to lower communication requirements and higher scalability. The iterative information exchange-based method can present actors more opportunity to exchange their operational conditions and willingness, but may need more time to reach equilibriums. In practical applications, the iterative information exchange-based method is expected to be useful in the scheduling phase, while the one-time information exchange-based method is more suitable of the real-time control phase.

\section{Literature review regarding application of transactive control in smart grids}

This section overviews the studies that apply the transactive control framework for managing distributed energy resources with different purposes in smart grids.

In [7], the transactive control method is applied to solve distribution network grid congestion between the distribution system operator and the electric vehicle (EV) fleet 

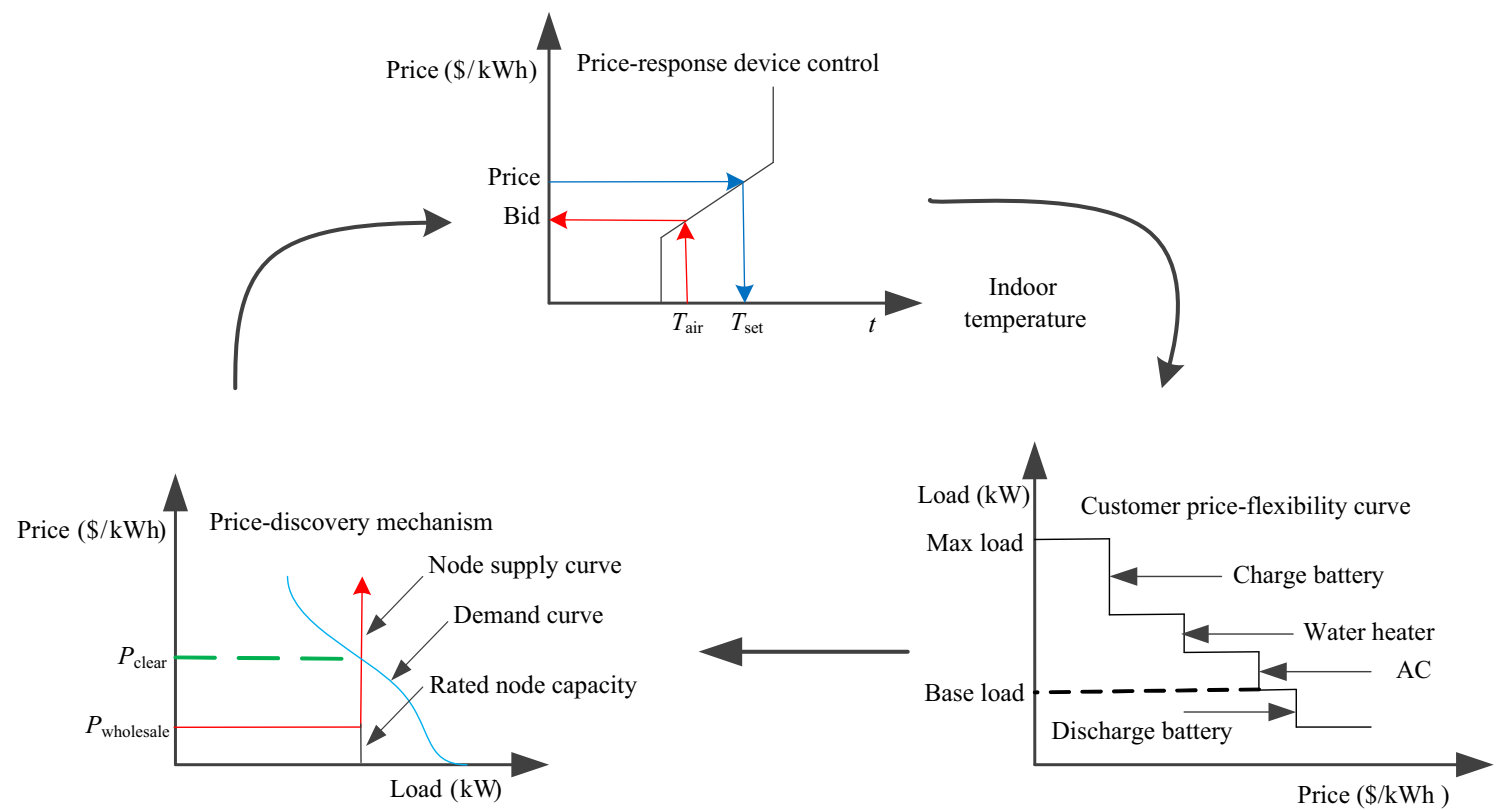

Fig. 2 Key aspects in one-time information exchange-based transactive control [33]

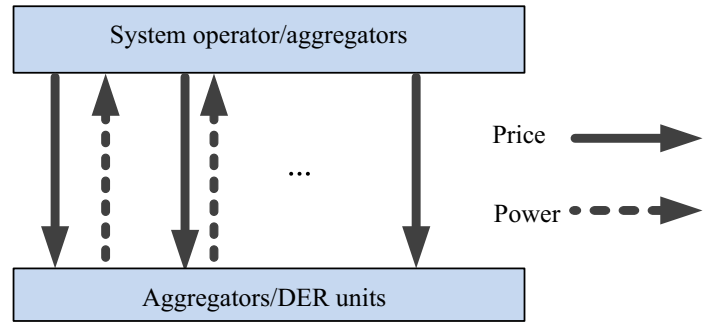

Fig. 3 Scheme of iterative information exchange-based method

operators, who manage the charging of EVs centrally. Firstly, the EV fleet operators (FO) generate optimal charging schedules, based on the energy spot price. However, the sum of the charging schedule of all the FOs may bring operational challenges to the DSO, and thus it needs to be modified. Then, a flexibility cost function of FO is formulated that reflects the charging power deviation from the scheduled charging power in the form of a quadratic function. The overall objective is to minimize the flexibility cost function of EV FOs with respect to the grid capacity constraints. The minimization is formulated as a Lagrange problem, and solved iteratively using a decomposition algorithm. The Lagrange multipliers are interpreted as congestion price that coordinates the EV FO's charging profiles. Furthermore, the study is extended in [21] to solve the voltage-band violations by introducing congestion prices at the buses level.

In [22], Ipakchi pointed out that a higher penetration of distributed energy resources will require greater attention to distribution congestion issues, and a need for improved distribution-automation and distribution-management capabilities. A transactive control approach is proposed to solve these problems. In the example described in the paper, a plug-in electric vehicle requests $7.8 \mathrm{kWh}$ of charging energy over the next two hours. This request can be presented as a demand transaction and sent to a demand-side management application operated by the utility. Knowing the transaction delivery point to which the car charger is connected, this application will check the available capacity of the low-voltage distribution transformer and feeders. Then it determines whether the additional load will impact the circuit reliability and cause any adverse phase imbalances. The demand-side management system will then schedule the charging for the requested time period. At the same time, the management system may receive many more charging requests that have to be checked and coordinated with wholesale scheduling at the substation supplying the feeders, to ensure adequate supply. Each of these actions could be modeled as a transaction among the consumers, the utility, and suppliers.

Besides the application of congestion and voltage management $[7,21,22]$ in the distribution system, a conceptual framework of using transactive control is proposed in [26] that aims to optimally coordinate the operation of self-interested individual decision-makers that emerge in the distribution system. The optimal operation of the grid is described according to a set of predefined technical and economic targets, and can be achieved by influencing the behaviors of the decision-makers with appropriate market signals. In the study, the authors argue that complex system theory can be used to support the framework. In addition, a 
multi-agent model is built in the study to test the transactive control strategy.

Reference [36] formulated a class of finite horizon dynamic game (or a transactive control system) to optimally control the charging profile of a large number of electric vehicles. Within the game, the control objective is to minimize electricity-generation costs by establishing an EV charging schedule that fills the overnight demand valley. Moreover, the paper established a sufficient condition under which the system converges to the unique Nash equilibrium. In order to implement the transactive control system, an iterative algorithm for computing the unique Nash equilibrium is proposed, which includes four steps:

Step 1: The utility broadcasts the forecast of base demand to all the EVs.

Step 2: Each of the EVs proposes an optimal charging strategy that minimizes its charging cost, with respect to a common aggregated EV demand broadcasted by the utility.

Step 3: The utility collects all the optimal charging strategies proposed by the individual EVs, and updates the aggregated EV demand to all EVs.

Step 4: Repeat Step 2 and Step 3 until the optimal strategies proposed by all EVs no longer change.

A similar study performed in [37] proved that a transactive control-based algorithm converges to optimal charging profiles, irrespective of the specifications of EVs, even with asynchronous computation. Besides, the authors also extended the algorithm to track a given load profile and to real-time implementation. Furthermore, in [38], the study deals with more loads in a multiple residence setup. The study finds the social-welfare maximization for energy scheduling, between a utility company and residential energy-users. The problem is solved by a distributed subgradient method that can be supported by an advanced metering infrastructure (a two-way communication network).

In addition to optimally managing the residential energy of a utility [36-38], the study in [29] suggested a two-stage residential energy-management method that considered network operational constraints. In the first stage, a dayahead pricing scheme and residential appliance scheduling are determined through the interaction of the utility company and residential customers. In the second stage, prices are updated based on the actual residential loads that draw lessons from the locational marginal price (LMP) used in the transmission system. In addition, in [30, 31], transactive control is used to coordinate the thermostatic loads with the purpose of realizing efficient energy allocation, subject to peak energy constraint (feeder capacity constraints described in the study).

In [24], a scalable three-step approach for demand-side management of EVs is presented. The three steps consist of aggregation, optimization, and control. In the aggregation step, individual EV charging constraints are aggregated upwards. In the optimization step, the aggregated constraints are used for the scalable computation of a collective charging plan, which minimizes costs for the electricity supply. In the real-time control step, the objective to divide the optimal power generated in Step 2 between the individual EVs, is determined by a marketbased priority scheme. The work is further developed in [25], where an event-driven dual coordination mechanism is presented at the real-time control level. The simulation result indicated that the number of messages exchanged with the EVs was significantly reduced, by at least $64 \%$. In [23], the transactive control framework is proposed to manage the charging of electric vehicles, and incorporates a distribution transformer and voltage constraints. A hierarchical multi-agent structure was used in the study, consisting of an auctioneer agent, substation agent, and EV device agent. The substation agent summed up the bid functions of all the underlying devices in a low-voltage network, and in turn sent the bid function to a unique auctioneer agent who defined the equilibrium price. In addition, the substation agent also ensured that the grid constraints were not violated, given the possible equilibrium price.

In [19], the auction-based transactive control is applied to control the cluster of loads with the purpose of providing spinning reserves. Firstly, each device defines a utility function for the utilization of the power flexibility; e.g., the corner price model developed in [24] is applied to calculate the bid function of an EV. Then, in real-time operation, all the device agents send their bids to a concentrator agent or fleet operator agent. The concentrator agent sums up the bid functions of their zone, and then sends the aggregated bid function to a unique auctioneer agent. Finally, the auctioneer agent will define the equilibrium price as the intersection of the aggregate bid functions and the supply bid function. After the equilibrium price is defined, it is sent back to all of the devices agents, and the corresponding power of the device agents will be determined. The market-clearing takes place every 15 minutes, or can be made event-driven. Furthermore, the transactive control method is extended to cooperate in frequency reserve markets.

In [20], a hierarchical transactive control architecture combines market transactions at the tertiary level with inter-area and unit-level control at the primary and secondary level. The purpose of the hierarchical transactive control is to ensure frequency regulating, using optimal allocation of resources in the presence of uncertainties in renewables and load. Models and controllers developed at the tertiary level follow the standard market-clearing procedure that aims at social-welfare maximization. The 
problem is solved iteratively, and the global asymptotic stability of the overall system is established.

In [27, 28], a novel day-ahead pool market mechanism is proposed, to facilitate the participation of flexible demand in the electricity market. In the study, the market-clearing optimization problem is, firstly, converted from a socialwelfare maximization problem to an equivalent generation cost-minimization problem, and then solved indirectly by its Lagrangian dual problem. The mathematical decomposition scheme is interpreted as a two-level iterative marketclearing mechanism, with the elements of lambda representing the 24-hour electricity price. The proposed market mechanism is demonstrated with electric vehicles and heatpump systems.

In summary, a key operational parameter used in transactive control is value (i.e., cost/utility functions in [7, 19, 21-25, 36, 37]). Thereafter the equilibrium price can be discovered and the transaction can be executed. It is seen that iterative information exchange is required to reach equilibriums between the fleet operator and electric vehicles in [7, 21, 36, 37], while only one-time information exchange is required in [19, 21-25] to reach the equilibrium. The aforementioned studies are summarized in Table 1.

\section{Demonstration projects in US and Europe}

In this section, four demonstration projects are briefly introduced: the GriseWise Olympic Peninsula project, the Pacific Northwest Demonstration project, and the GridSMART demonstration project from the USA; and the Powermatching City project from Europe. The summary of demonstration project using transactive control framework is shown in Table 2.

GridWise Olympic Peninsula project [39]: The project adopted transactive control to coordinate the power use of residential electric water heaters and thermostats, commercial building-space conditioning, municipal water pump loads, and several distributed generators, with the purpose of reducing stress on local distribution networks.

The field demonstration took place in Washington and Oregon, and was paid for by the U.S. Department of Energy and several northwest utilities. Real-time price at 5-minute intervals was found to be an effective control signal for managing distribution congestion. Peak loads were effectively reduced on the experimental feeder.

The Pacific Northwest Demonstration project [40]: The project expanded upon the experience of the previously described GridWise Olympic Peninsula project. This project provided two-way communication between distributed generation, storage, and demand assets, and the existing gird infrastructure. The purpose was to use and test the tranactive control signal. The signals communicated the cost of delivering energy to a specific location. Using automated controller, the devices such as water heaters, electric furnaces etc. could make their own decision when to use electricity.

GridSMART demonstration project [41]: The project aimed to design, build, and operate a transactive control system, to engage residential consumers and their end-use resources, to address the local-scale grid congestion. The method has the advantage of providing greater efficiency under normal operating conditions, and greater flexibility to react under situations of system stress. Three main aspects were studied: the impact on system operations, and on households, and observations about the sensitivity of load to price changes.

Powermatching City [42]: PowerMatching City is a living lab environment based on state-of-the-art off-theshelf consumer products that have been altered to provide flexibility, and allow coordination with the smart grid [43]. The technology used in the project is PowerMatcher [44]; a 'demand response' technology that balances all smart devices, from low voltage to high voltage, in a virtual

Table 1 Summary of references using transactive control framework

\begin{tabular}{lll}
\hline References & Operational purpose & Implemention methods \\
\hline$[7,21,23]$ & Congestion and voltage management & Iterative information exchange based \\
{$[19]$} & Secondary frequency control & One-time information exchange based \\
{$[22]$} & Congestion management in distribution network & Not specified \\
{$[26]$} & Distribution system general operation & Iterative information exchange based \\
{$[36-38]$} & Residential optimal energy management of utility & Iterative information exchange based. \\
{$[29]$} & Residential optimal energy management of utility & considering network operational constraints \\
{$[30,31]$} & Residential optimal energy management of utility & Iterative information exchange based \\
{$[24,25]$} & Mansidering network operational constraints & One-time information exchange based \\
{$[20]$} & Tertiary frequency control & One-time information exchange based \\
{$[27,28]$} & New electricity spot market mechanism & Iterative information exchange based \\
\hline
\end{tabular}


Table 2 Summary of demonstration project using transactive control framework

\begin{tabular}{llc}
\hline Projects & Operational purpose & Implemention methods \\
\hline GridWise Olympic Peninsula project & Congestion management & $\begin{array}{c}\text { One-time information } \\
\text { exchange }\end{array}$ \\
$\begin{array}{l}\text { The Pacific Northwest Demonstration } \\
\text { project }\end{array}$ & $\begin{array}{c}\text { Coordinate the operational decision of distributed energy } \\
\text { resources }\end{array}$ & $\begin{array}{c}\text { One-time information } \\
\text { exchange }\end{array}$ \\
$\begin{array}{ll}\text { GridSMART demonstration project } \\
\text { Congestion management }\end{array}$ & Manage the balance between supply and demand & $\begin{array}{c}\text { One-time information } \\
\text { exchange }\end{array}$ \\
& & $\begin{array}{c}\text { One-time information } \\
\text { exchange }\end{array}$ \\
\hline
\end{tabular}

market [15]. In the first phase of the demonstration, PowerMatching City consisted of 22 common Dutch households, located near the city of Groningen, in the Netherlands. Later, this number was scaled up further. The houses were fitted with either a domestic combined heat and power unit (micro-CHP) or a heat pump with a gasfired heater [45]. Some households also contained intelligent white-good appliances, and electric vehicles were integrated as well. Outside the district, a 2.5 MW wind turbine was available. The output power of the wind turbine could be scaled down digitally to match the consumption of the households. All devices were interfaced with PowerMatcher software, to operate PowerMatching City as a virtual power plant (VPP). It was concluded that the VPP successfully followed its optimized energy profile, and provided the required regulatory power at the same time [46].

\section{Case studies}

As the market adoption of DER reaches regional scale it will create significant issues in the management of the distribution system related to existing planning and control. This is likely to lead to problems for power quality and reliability, since integrating distributed resources into wholesale markets, without aligning distribution control schemes, may create unacceptable consequences. Furthermore, as discussed in [10], as distributed energy resources increase, the need to balance these resources across the distribution system will likely give rise to the development of a distribution system control tier, to complement the bulk power system control tier.

Thus, in this study, we present two relevant case studies that apply transactive control framework at the distribution system level. The first case gives a brief overview of the developed work [7], where distribution congestions are solved by a transactive control framework. In the second case, a transactive control is applied to manage the distribution system balance dynamically between supply and demand.
To apply the transactive control in both case studies, a software agent (either an aggregator in Section 5.1 or a local device agent in Section 5.2) will be present to represent the DER's operational flexibility. The agent will form a cost function (Section 5.1) or a Walrasian demand function (Section 5.2) to characterize the flexibility. These functions are bid into a virtual market where the equilibrium will be found among the agents. The conditions of the equilibrium's existence depend on the convexity of the cost functions or the monotonicity of the demand functions. More assumptions and conditions of applying transactive control method are presented in Section 6.

\subsection{Distribution grid congestion management considering electric vehicle integration}

In this case, we use electric vehicles as an example to be integrated in the distribution system. Figure 4 shows that the system consists of three actors: distribution system operator, aggregators, and electric vehicle owners located in a low-voltage network.

In this system, we assume aggregator manage the charging schedule of electric vehicles centrally, with the purpose of procuring the electricity from a spot market in a low-price period, and fulling the energy requirements of EV charging. However, if all the EVs are charged in the low-price period, the aggregated power may introduce a congestion problem into the distribution network. Thus, transactive control will be used between the DSO and the aggregator to coordinate the power schedules. In [7] and [21], a flexibility cost function that represents the cost of the power preference difference of aggregators in each time slot $t$ is proposed as follows.

$\mu_{k}=C_{k, t}\left(\tilde{P}_{\mathrm{Agg}}^{k}(t)-P_{\mathrm{Agg}}^{k}(t)\right)^{2}$

subject to

$\sum_{t=1}^{T} \tilde{P}_{\mathrm{Agg}}^{k}(t) n_{t}=\sum_{t=1}^{T} P_{\mathrm{Agg}}^{k}(t) n_{t}$ 


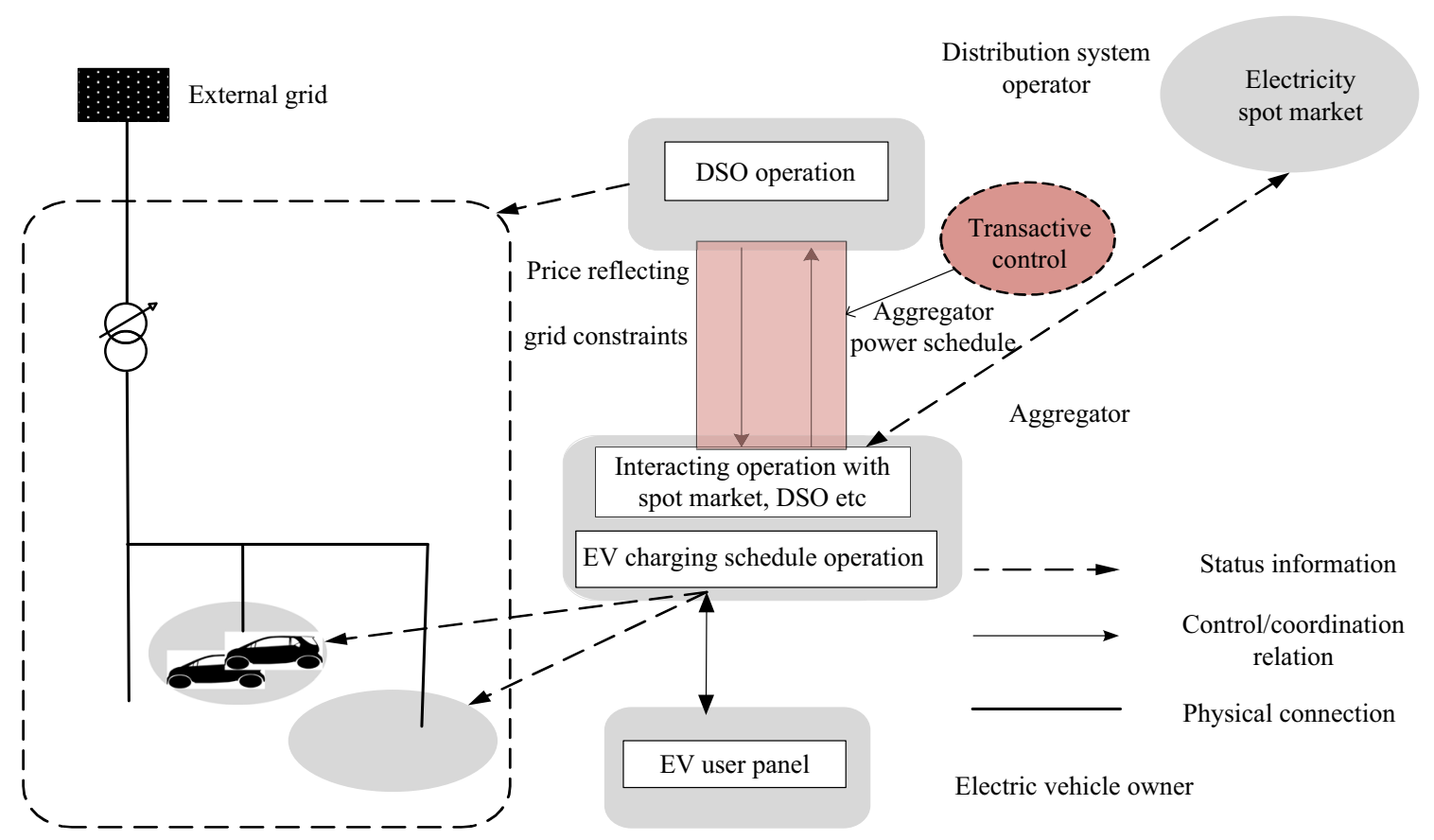

Fig. 4 Transactive control system for electric vehicle integration

where $\tilde{P}_{\mathrm{Agg}}^{k}$ is the control variable; $P_{\mathrm{Agg}}^{k}(t)$ is the preferred schedule; $C_{k, t}$ is the weighting factors that are associated with the power difference; $n_{t}$ is the length of time slot $t ; k$ is the index of the aggregator (Agg).

The objective is to minimize the cost of all the aggregators with respect to the power-transformer constraint from the DSO.

$\min \sum_{k=1}^{N_{\mathrm{Agg}}} \sum_{t=1}^{T} C_{k, t}\left(\tilde{P}_{\mathrm{Agg}}^{k}(t)-P_{\mathrm{Agg}}^{k}(t)\right)^{2}$

subject to

$\sum_{k=1}^{N_{\mathrm{Agg}}} \tilde{P}_{\mathrm{Agg}}^{k}(t) \leq P_{\text {Tran }}^{\mathrm{Max}}(t)$

$\sum_{t=1}^{T} \tilde{P}_{\mathrm{Agg}}^{k}(t) n_{t}=\sum_{t=1}^{T} P_{\mathrm{Agg}}^{k}(t) n_{t}$

where $P_{\mathrm{Tran}}^{\mathrm{Max}}$ is the power-transformer capacity. By introducing Lagrange multipliers or shadow price $\lambda(t)$, and considering the constraint (5), problem (3) is transferred into a partial Lagrangian problem.

$$
\begin{gathered}
\sum_{k=1}^{N_{\mathrm{Agg}}} \sum_{t=1}^{T} C_{k, t}\left(\tilde{P}_{\mathrm{Agg}}^{k}(t)-P_{\mathrm{Agg}}^{k}(t)\right)^{2}+\sum_{t=1}^{n_{t}}(t) \lambda(t) \\
\cdot\left(\sum_{k=1}^{N_{\mathrm{Agg}}} \tilde{P}_{\mathrm{Agg}}^{k}(t)-P_{\operatorname{Tran}}^{\operatorname{Max}}(t)\right)
\end{gathered}
$$

Problem (6) can be broken down for each aggregator given the $\lambda^{\omega}(t)$ in each iteration. Thus each aggregator will get a new optimal schedule denoted as $P_{\mathrm{Agg}}^{k}\left(t, \lambda^{\omega}(t)\right)$, and the Lagrange multipliers are updated according to $\lambda^{\omega+1}(t)=\lambda^{\omega}(t)+\alpha_{\omega}\left(\sum_{k=1}^{N_{\mathrm{Agg}}} P_{\mathrm{Agg}}^{k}\left(t, \lambda^{\omega}(t)\right)-P_{\mathrm{Tran}}^{\mathrm{Max}}(t)\right)$ until the shadow price convergence. $\omega$ is the index of the negotiation step; $\alpha_{\omega}$ is the stepsize and normally it is a constant. The converged shadow price $\lambda^{\omega+1}(t)$ is used as the congestion price.

To illustrate the modeling method, we assume 72 households are connected to a power transformer, and the power transformer allocates $200 \mathrm{~kW}$ to two EV aggregators. Each aggregator (Agg 1 and Agg 2) has $36 \mathrm{EVs}$, which means a $100 \%$ penetration case. The scheduling period considered in the study starts from 16:00 to 06:00 and a 15-minute interval is used ( 56 time slots are defined). The hourly predicted dayahead market price is assumed to be known by the aggregator. Each aggregator uses a linear programming method to generate the aggregated power schedule. For the parameters of the EVs used in generating charging schedule, the battery capacity of each EV is set to $24 \mathrm{kWh}$, the initial state of charge of individual EV is set to 0.2 of the battery capacity, and the maximum charging power is limited to $3.7 \mathrm{~kW}$, which fits with the Danish case (16 A, $230 \mathrm{~V}$ connection). With the values of these parameters, the aggregated power of Agg 1 and Agg 2 is shown in Fig. 5.

We note that the aggregated power schedule during time slots 45 to 48 , i.e., 02:00 to 03:00, exceeds the allocated capacity of the transformer. Therefore, the power schedule at these four time slots needs to be modified by the method 


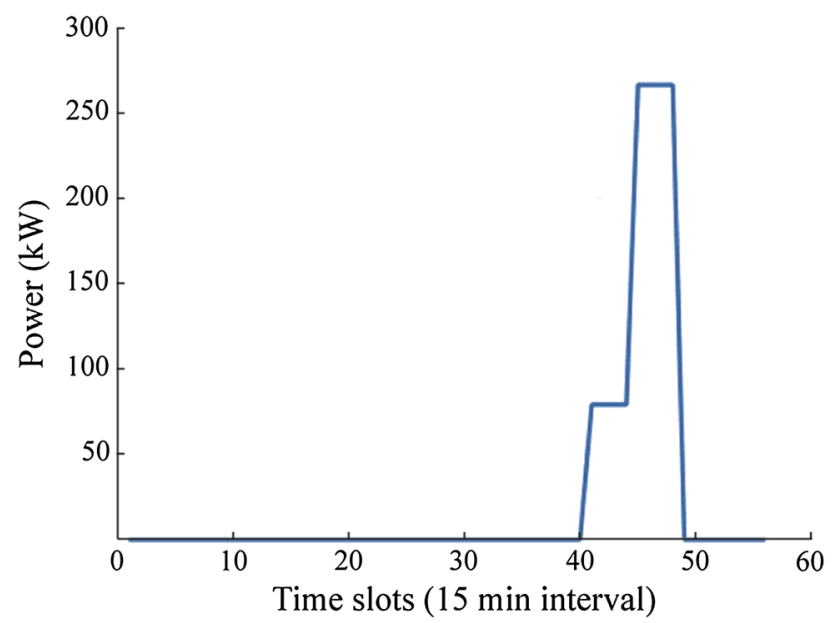

Fig. 5 Aggregated power schedule of Agg 1 and Agg 2

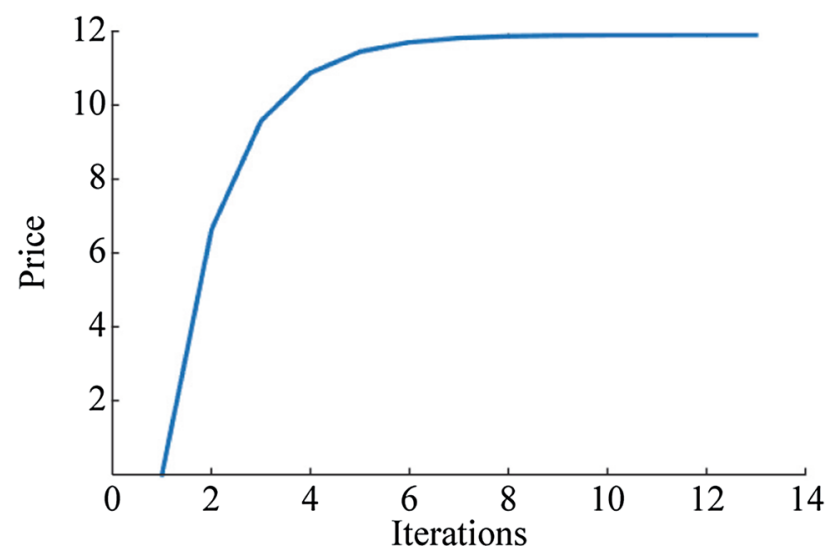

Fig. 6 Convergence of shadow price

described in this section. The weighting factor rates $C_{1, t}$ and $C_{2, t}$ in (1) are set to 0.5 and 0.1. A constant stepsize $\alpha_{\omega}=0.1$ is chosen for the Lagrangian multiplier update. With the values of the parameters, Figs. 6 and 7 show the convergence of the price and the aggregators at time 02:00 to 03:00. As noted in Fig. 7, the sum of the power schedule of Agg 1 and Agg 2 is $200 \mathrm{~kW}$ after the iterative information exchange. The simulation is performed within MATLAB using CVX, a package for specifying and solving convex programs [47].

\subsection{System balance}

In this section, the PowerMatcher development is described, to show the application of the transactive control framework for system balance. PowerMatcher has been designed as a general-purpose coordination mechanism for balancing demand and supply in large clusters of distributed generation, demand response, and electricity storage connected to the distribution grid, and is open-source available. The open-source reference implementation of

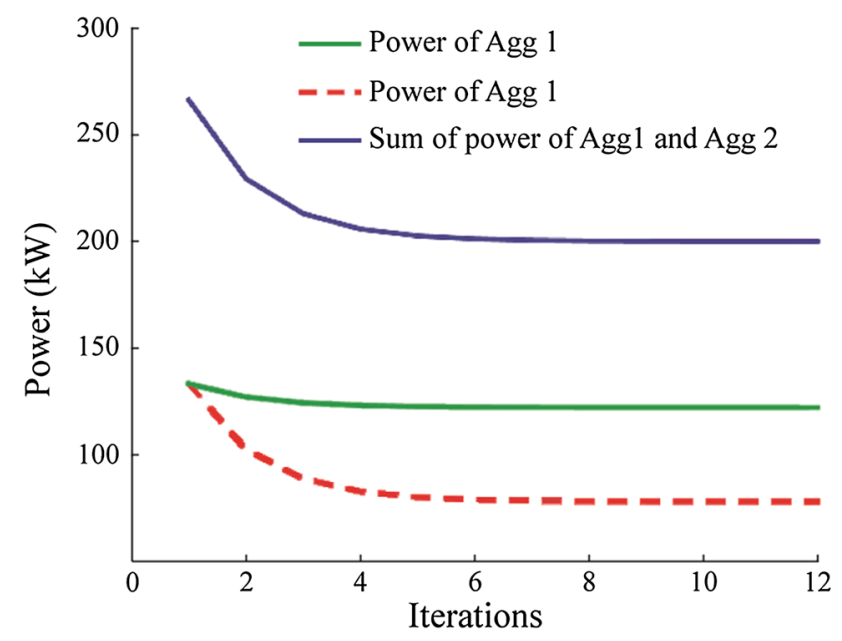

Fig. 7 Convergence of power schedule of Agg 1 and Agg 2

PowerMatcher is programmed in Java which makes it deployable on a wide variety of processing platforms. The local device control agent software makes use of OSGi, which implements an open and modular Internet-of-Things architecture for Java. This makes the PowerMatcher software easily deployable and maintainable. PowerMatcher $[43,48,49]$ has been shown to improve the match between consumption and production by: (1) adding value to renewable/distributed generation and demand-side flexibility; (2) improving the integration of renewable generation; (3) mitigating congestion (i.e. local network overloading) in distribution networks. A comprehensive treatment of this technology can be found in [50].

PowerMatcher is based on multi-agent systems technology. Within a PowerMatch cluster, the agents are organized into a logic tree. The leaves of this tree are a number of local device agents. The root of the tree is formed by the auctioneer agent, which handles the price forming by searching for the equilibrium price. In order to obtain scalability, concentrator agents can be added to the structure as tree nodes. A local device agent is representative of a DER device. For a DER unit to be able to participate in a PowerMatcher cluster, its associated agent must communicate its momentary bid curve or demand function to the auctioneer agent. This function defines the DER's electricity demand $d(p)$ for a given price $p$. An offer to produce a certain amount of electricity against a certain price is expressed by negative $d(p)$ value. The core functionality of the auctioneer and the concentrator is to run the electronic market, allocating the electrical power resource to the local device agents. The electronic market solves this allocation problem by finding the general equilibrium price $p^{*}$ such that:

$\sum_{a=1}^{N_{\mathrm{a}}} d_{a}\left(p^{*}\right)=0$ 
where $N_{\mathrm{a}}$ is the number of local device agents; $d_{a}(p)$ is the demand function of agent $a$ that states the agent's demand or supply at a given price $p$.

A number of larger-scale demonstration projects have been conducted successfully with the PowerMatcher technology, to demonstrate its feasibility and potential. Here, we zoom in on one of those, Couperus Smart Grid, alongside the PowerMatching City project described in Section 4 . The Couperus project demonstrated the ability to perform tasks concurrently for system-wide balancing and local distribution-level congestion management. In this text we focus on the balancing aspect.

The Couperus Smart Grid project turned an apartment block, with approx. Three hundred apartments and individual heat pumps for heating, into a Virtual Power Plant using PowerMatcher technology, performing both imbalance reduction and capacity management (peak shaving) in the local LV-to-MV (low voltage to medium voltage) substation. The operational flexibility can be used to optimize the trading position of the energy supplier involved; for example, by reducing the imbalance of a wind farm; i.e. the difference between the day-ahead forecast and the actual production. A more detailed description of the project's outcomes can be found in [51], of which we give an overview in this section.

Each of the apartments in the building is heated by a heat pump with a nominal electrical power of $1 \mathrm{~kW}$. The systems are used for space heating and tap-water heating, and are equipped with a heat-storage tank. The inlet water for all heat pumps comes from a groundwater heat exchanger (aquifer) providing a low inlet temperature, leading to a high energy efficiency of the heating process.

The balancing functionality of the VPP is performed by the so-called Imbalance Agent. In the case of this project, this software agent used the flexibility of the heat pumps to reduce the imbalance of the wind turbine farm. The realtime imbalance of the wind farm was used as an input for this agent. During this part of the test, 150 heat pumps were connected to the VPP. As each heat pump has a nominal power of $1 \mathrm{~kW}$, and a low duty cycle (i.e. the heat pumps operate with relatively long off times), the power deviations requested from the VPP were chosen in the range of $\pm 10 \mathrm{~kW}$. From the energy supplier in the project, the imbalance signal of a nearby wind turbine was fed into the system, scaled to this response range. The imbalance signal was the difference between the day-ahead forecasted wind generation and the actual generation.

To reduce the wind power imbalance, the imbalance agent bids the imbalance signal into the electronic market. In case of a surplus of wind power, the price on the electronic market to go down, resulting in a number of heat

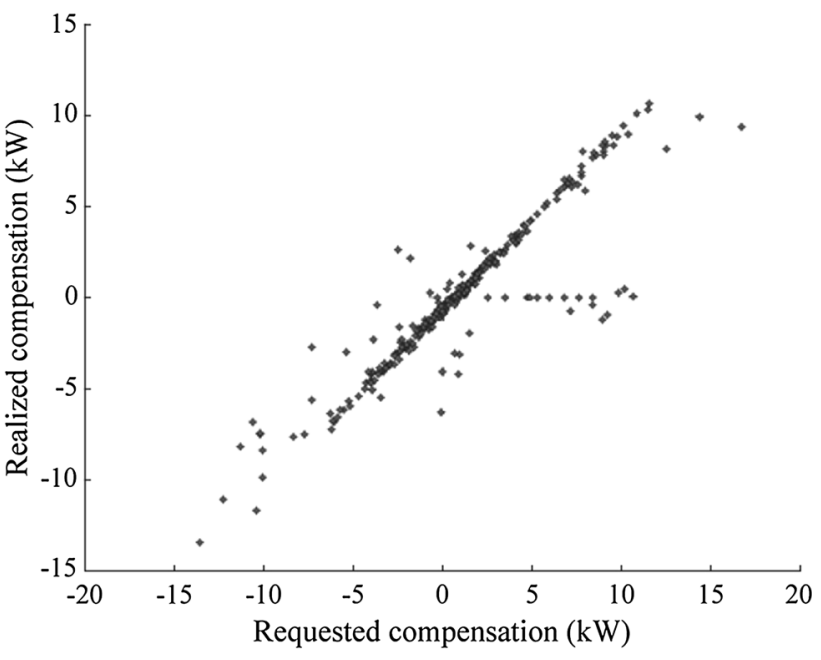

Fig. 8 Relation between requested and realized imbalance reduction over two winter weeks [51]

pumps to switch on to consume the surplus. Similarly, in case of underproduction, the price will rise and electricity demand from the heat pumps will fall.

Figure 8 shows the relation between the requested and realized imbalance reduction, as delivered by the VPP over two weeks in the winter. On almost all occasions, the compensation requested can be fulfilled quantitatively: both signals are highly correlated (correlation equals 0.90 ) with a slope equal to 1 . The response accuracy in the figure is $94.6 \%$, indicating that the PowerMatcher performs well coordinating among the heat pumps to compensate the unexpected under- or overproduction of the wind turbine. Note that during the winter the heat pumps have the highest running time, providing heat to the apartments, which limits their response. Thus, these results can be seen as a worse-case category performance.

\section{Research challenges}

Compared to conventional power systems operation, one of the important advantages of transactive control is the incorporation of DER users' priorities/ needs/ utilities/ costs into the operation of the power systems. Thus the entire system can achieve an optimum balance necessary to meet objectives and constraints. Therefore, it is important to investigate the price-response behavior of DERs and to design the optimal pricing strategy. Next to these two challenges, another issue is how to create and operate a market where efficiency and transparency are guaranteed. On the method front, how to ensure the convergence of the transactive control application, and increase the speed of the convergences, are also important issues. In addition, the requirements of the ICT infrastructure for communication 
among various stakeholders are another important challenge. Last but not least, the standardization of interface of transactive energy is essential for successful implementation. Besides these general challenges, specifically, regarding application of transactive control in solving network problems, more studies are needed on how to calculate the base line, and how to use the 'shadow price' in practice.

\subsection{Price-response behavior of DERs}

In a transactive control framework, it is necessary for the DER owner to be price sensitive, and able to actively respond to the price informed by the system operator. However, it is a challenge to model the price-response behavior of customers properly. Both [52] and [53] describe how, if most of the buildings in a distribution circuit have an energy-management system that turns up the air-conditioning or the heating when electricity prices drop below a threshold, then the distribution circuit, and indeed the entire grid, can be destabilized, due to the amplified spikes. To understand the price-response behavior of customers, methods including a conditional logit model [54] and a parametric stochastic process [55] are presented in the literature. Nevertheless, it is noted in $[54,55]$, the extent to which a properly designed price signal could assist in maintaining that grid reliability will remain open until the DER owner's price responsiveness is tested empirically through experiments.

\subsection{Pricing strategy design and convergence issue}

As discussed in [52], a simple price strategy may destabilize the power system operation. In contrast to conventional power system operation, in the near future, more distributed energy resources integrated into operation requires additional research on the convergence of dynamic pricing. In real-time operation, new market mechanisms are needed to create effective interactions that are closely linked to the distribution control systems and DERs, to ensure the security and efficiency of the system, as well as the optimality of the DER operation. Regarding the convergence of the iterative-based method under the circumstance of transactive control, [29] and [38] discuss that, in general, the convergence can be obtained by following certain stepsize rules. In addition, as described in [38], the algorithms find near-optimal schedules, even when advanced metering infrastructures (AMI) messages (updated prices and residential load) are lost, which can happen in the presence of malfunctions, or noise in the communication network. However, much research is needed to increase the convergence speed, since high iteration numbers are seen in many studies, and these imply more time is needed for convergence.

\subsection{Market place design}

To ensure enough competition and fairness in the capacity market, one prerequisite is the number of market participants; e.g., FOs in Section 5.1. If there are few FOs in the distribution area, and issue such as market power will become a major challenge from the market perspective. Thus how to define the market rules that can create enough competition and fairness will be a big challenge in transactive control application.

It is worth mentioning that the application of transactive control does not call for a central market. A centralized marketplace can potentially bring too many participants, considering DERs' direct participation, into one platform, which brings challenges both for market design and for operation. The creation of distributed marketplaces may be a reasonable approach to involve more DERs into the current operation. The distributed marketplaces could possibly following the existing geographical distribution areas.

\subsection{Requirements for ICT infrastructure}

In addition to conventional stakeholders, the new stakeholders in smart grid will probably include aggregators and prosumers. Thus communication will be an important issue, since a large population of stakeholders needs to be connected. For example, in the case presented in Section 5.1, FOs need to communicate well with EVs, in order to make an optimal charging schedule, and this information includes driving pattern, state of charge, and some other preferences of EV owners. The time-constraint is not an essential issue in the scheduling problem, although FOs need real-time information exchange to ensure the schedule is properly executed. For the interaction between FOs and the distribution grid capacity market operator, real-time communication is a challenge, but a reasonable time range can be established, as well as trying to limit the market iteration with certain rules. This kind of setup will require an advanced ICT infrastructure that is becoming affordable.

Furthermore, as discussed in [45], an ICT delay during market-clearing can introduce an instability issue into the power system. It is noted in the paper that there is a counterintuitive relationship between the market-clearing time and the price-signal delay: when the market-clearing time is relatively long, delaying the price signal can improve the market's stability, while reducing the communication delay can destabilize the market. This counterintuitive effect shows that the full impact of information technology on power markets can be significant and difficult to anticipate. Therefore, as markets are incorporated into transactive control applications, careful attention 
should be paid to the effects of information technology on the market's dynamic behavior.

\subsection{Standardization of interface of transactive energy}

The envisioned standardized interfaces include two aspects: (1) an interface for all the actors participating in the transactive energy market, which will define the bid forms; e.g., according to the market structure, market operating mechanism; (2) an interface with DERs; i.e., the information sent by the market operator should be understood by the DERs, and thus the DERs can respond to the signal. To build a standardized platform that accelerates the development of transactive energy, several initiatives have been seen in the field, including: USEF (universal smart energy framework [56]); openADR (open automated demand response) alliance [57]; and EF-PI (Energy Flexibility Platform and Interface) white paper [58].

\subsection{Baseline issue}

The baseline is normally defined as an estimate of the electricity that would have been consumed by a customer in the absence of a demand-response event $[59,60]$. For example, in the first case study, the transactive control method enables the demand response. The aggregator that optimally generates the energy schedule is an operation in the absence of a demand-response event. Thus, the optimized energy schedule of the aggregators will be used as a baseline in the transactive control method, to prevent grid constraint violation. However, aggregators may not make the energy schedule optimal. Instead, they can procure the electricity based on the customer's power usage pattern. Under this circumstance, the customer's normal daily profiles will be used as a baseline. Nevertheless, more research is needed to characterize the baseline, since it will be very important in the settlement stage.

\section{Conclusions}

In summary, from a demand response-enabling approach to a power system operational principle, the transactive control framework has shown its distinctive capability to incorporate DER users' priorities, needs, or costs that accommodate the high penetration of distributed energy resources smoothly into future power systems.

The implementation methods of transactive control include a one-time information exchange-based approach, and an iterative information exchange-based method. These two methods have been used widely in the literature and demonstration projects. Note that, for a hierarchical transactive control system, it is possible to combine both implementation methods, such as using the one-time information method on lower levels, and having an iterative information exchange method in the upper levels. Finally, there is also a need for policymakers to better understand the relationship between pricing schemes and control systems, as it relates to distributed energy resources to ensure proper market structures and rules, to maintain a highly reliable and clean energy system.

The current development trend is to integrate all the energy networks together, to hedge against the intermittency brought about by the increasing penetration of renewable energy. The ultimate goal of this development would be a so-called "Energy Internet", where energy can be polled and sent from anyone, anywhere, at any time. The operation of such a system would involve more participants from different energy networks than the ones seen today. The incentives for participation would be from some designed market mechanisms and eventually, the system operation would be based on handling various energy offers and requests from all the participants. In the operation, all the participants, including the grid operators, will come with their own objectives, as well as constraints, for participation. The problem will become highly complex, including concerns from both economic and technical aspects. Such situations may be suitable for the application of transactive control principles.

Acknowledgement The authors thank Pamela Macdougall of TNO, The Netherlands for her valuable comments on the draft of this article. Koen Kok's contribution to the writing of this article was financed by the TNO Early Research Program on Energy Storage and Conversion (ERP ECS) through the SOSENS project. This work is partly supported by the Danish iPower project (http://www.ipowernet. $\mathrm{dk} /$ ) funded by the Danish Agency for Research and Innovation (No. 0603-00435B).

Open Access This article is distributed under the terms of the Creative Commons Attribution 4.0 International License (http:// creativecommons.org/licenses/by/4.0/), which permits unrestricted use, distribution, and reproduction in any medium, provided you give appropriate credit to the original author(s) and the source, provide a link to the Creative Commons license, and indicate if changes were made.

\section{References}

[1] Medium-term renewable energy market report 2015: market analysis and forecasts to 2020. International Energy Agency (IEA), Paris, 2015

[2] Pineda I, Wilkes J (2015) Wind in power: 2014 European statistics. The European Wind Energy Association (EWEA), Brussels

[3] Ge SY, Xu L, Liu H et al (2015) Low-carbon benefit analysis on DG penetration distribution system. J Mod Power Syst Clean Energy 3(1):139-148. doi:10.1007/s40565-015-0097-z 
[4] Coster EJ, Myrzik JMA, Kruimer B et al (2011) Integration issues of distributed generation in distribution grids. PIEEE 99(1):28-39

[5] Pepermans G, Driesen J, Haeseldonckx D et al (2005) Distributed generation: definition, benefits and issues. Energy Policy 33(6):787-798

[6] Ela E, Milligan M, Kirby B (2011) Operating reserves and variable generation. NREL/TP-5500-51978. National Renewable Energy Laboratory (NREL), Golden

[7] Hu JJ, You S, Lind M et al (2014) Coordinated charging of electric vehicle for congestion prevention in the distribution grid. IEEE Trans Smart Grid 5(2):703-711

[8] De Martini P, Chandy KM, Fromer NA (2012) Grid 2020: towards a policy of renewable and distributed energy resources. Resnick Institute, California Institute of Technology, Pasadena

[9] Juamperez M, Yang G, Kjær SB (2014) Voltage regulation in LV grids by coordinated volt-var control strategies. J Mod Power Syst Clean Energy 2(4):319-328. doi:10.1007/s40565014-0072-0

[10] Hanninen S (2015) Detailed requirements and constraints for the control of flexibility. European FP 7 project ELECTRA (European Liaison on electricity committed towards long-term research activities for smart grids), ENGIE Lab Laborelec, Linkebeek

[11] Hansen H, Holm-Hansen HH, Samuelsson O, et al (2013) Coordination of system needs and provision of services. In: Proceedings of the 22nd international conference and exhibition on electricity distribution (CIRED'13), Stockholm, 10-13 June 2013, 4 pp

[12] Ding Y, Hansen LH, Cajar PD et al (2013) Development of a DSO-market on flexibility services. iPower WP3.8. iPower Consortium, Copenhagen

[13] Zegers AA, Brunner H (2014) TSO-DSO interaction: an overview of current interaction between transmission and distribution system operators and an assessment of their cooperation in smart grids. International Smart Grid Action Network (ISAGN) Discussion Paper Annex 6 Power T\&D Systems, Task 5. ISAGN, Paris

[14] GridWise transactive energy framework, Version 1.0. PNNL22946 Ver1.0. The GridWise Architecture Council, Richland, 2015

[15] Kok K, Warmer CJ, Kamphuis IG (2005) PowerMatcher: multiagent control in the electricity infrastructure. In: Proceedings of the 4th international joint conference on autonomous agents and multiagent systems (AAMAS'05), Utrecht, 25-29 July 2005, pp 75-82

[16] Hammerstrom D, Oliver T, Melton R et al (2009) Standardization of a hierarchical transactive control system. In: Proceedings of the grid-interop conference, Denver, 17-19 Nov 2009, pp 35-41

[17] Kok K, Widergren S (2016) A society of devices: integrating intelligent distributed resources with transactive energy. IEEE Power Energy Mag 14(3):34-45

[18] Nord Pool. http://www.nordpoolspot.com/About-us/

[19] Weckx S, D'Hulst R, Driesen J (2015) Primary and secondary frequency support by a multi-agent demand control system. IEEE Trans Power Syst 30(3):1394-1404

[20] Bejestani AK, Annaswamy A, Samad T (2014) A hierarchical transactive control architecture for renewables integration in smart grids: analytical modeling and stability. IEEE Trans Smart Grid 5(4):2054-2065

[21] Hu JJ, Yang GY, Bindner HW (2015) Network constrained transactive control for electric vehicles integration. In: Proceedings of the 2015 IEEE Power and Energy Society general meeting, Denver, 26-30 July 2015, 5 pp
[22] Ipakchi A (2011) Demand side and distributed resource management $-\mathrm{a}$ transactive solution. In: Proceedings of the 2011 IEEE Power and Energy Society general meeting, San Diego, 24-29 July 2011, 8 pp

[23] Weckx S, D’Hulst R, Claessens B et al (2014) Multiagent charging of electric vehicles respecting distribution transformer loading and voltage limits. IEEE Trans Smart Grid 5(6):2857-2867

[24] Vandael S, Claessens B, Hommelberg M et al (2013) A scalable three-step approach for demand side management of plug-in hybrid vehicles. IEEE Trans Smart Grid 4(2):720-728

[25] De Craemer K, Vandael S, Claessens B et al (2014) An eventdriven dual coordination mechanism for demand side management of PHEVs. IEEE Trans Smart Grid 5(2):751-760

[26] Bompard EF, Han B (2013) Market-based control in emerging distribution system operation. IEEE Trans Power Deliv 28(4):2373-2382

[27] Papadaskalopoulos D, Strbac G, Mancarella P et al (2013) Decentralized participation of flexible demand in electricity markets-part I: market mechanism. IEEE Trans Power Syst 28(4):3658-3666

[28] Papadaskalopoulos D, Strbac G, Mancarella P et al (2013) Decentralized participation of flexible demand in electricity markets-part II: application with electric vehicles and heat pump systems. IEEE Trans Power Syst 28(4):3667-3674

[29] Moradzadeh B, Tomsovic K (2013) Two-stage residential energy management considering network operational constraints. IEEE Trans Smart Grid 4(4):2339-2346

[30] Li S, Zhang W, Lian JM et al (2016) Market-based coordination of thermostatically controlled loads-part I: a mechanism. IEEE Trans Power Syst 31(2):1170-1178

[31] Li S, Zhang W, Lian JM et al (2016) Market-based coordination of thermostatically controlled loads-part II: unknown parameters. IEEE Trans Power Syst 31(2):1179-1187

[32] Barrager S, Cazalet E (2014) Transactive energy: a sustainable business and regulatory model for electricity. Baker Street Publishing, San Francisco

[33] Hu JJ (2014) Control strategies for power distribution networks with electric vehicle integration. Ph.D. Thesis. Technical University of Denmark, Copenhagen

[34] Disfani VR, Fan LL, Piyasinghe L et al (2014) Multi-agent control of community and utility using Lagrangian relaxation based dual decomposition. Electr Power Syst Res 110:45-54

[35] Kraning M, Chu E, Lavaei J et al (2013) Dynamic network energy management via proximal message passing. Found Trend Optim 1(2):70-122

[36] Ma ZL, Callaway DS, Hiskens IA (2013) Decentralized charging control of large populations of plug-in electric vehicles. IEEE Trans Control Syst Technol 21(1):67-78

[37] Gan L, Topcu U, Low SH (2013) Optimal decentralized protocol for electric vehicle charging. IEEE Trans Power Syst 28(2):940-951

[38] Gatsis N, Giannakis GB (2012) Residential load control: distributed scheduling and convergence with lost AMI messages. IEEE Trans Smart Grid 3(2):770-786

[39] Katipamula S, Chassin DP, Hatley DD, et al (2006) Transactive controls: market-based GridWiseTM controls for building systems. PNNL-15921. Pacific Northwest National Laboratory, Richland

[40] Pacific Northwest Smart Grid Demonstration Project. http:// www.pnwsmartgrid.org/

[41] Widergren S, Subbarao K, Fuller J et al (2014) AEP Ohio gridSMART demonstration project real-time pricing demonstration analysis. PNNL-23192. Pacific Northwest National Laboratory, Richland

[42] PowerMatching City. http://www.powermatchingcity.nl 
[43] Kok K, Roossien B, MacDougall P et al (2012) Dynamic pricing by scalable energy management systems: field experiences and simulation results using PowerMatcher. In: Proceedings of the 2012 IEEE Power and Energy Society general meeting, San Diego, 22-26 July 2012, 8 pp

[44] The suite. PowerMatching Suite. http://flexiblepower.github.io/ technology/powermatcher/

[45] Roossien B, MacDougall PA, Van den Noort A (2010) Intelligent heating systems in households for smart grid applications. In: Proceedings of the 2nd international conference on innovation for sustainable production (I-SUP'10), Bruges, 18-21 April 2010

[46] Roossien B, Van den Noort A, Kamphuis R, et al (2011) Balancing wind power fluctuations with a domestic virtual power plant in Europe's first smart grid. In: Proceedings of the 2011 IEEE Trondheim PowerTech conference, Trondheim, 19-23 June 2011, 5 pp

[47] Grant M, Boyd SP (2013) CVX: MATLAB software for disciplined convex programming, version 2.0 beta. CVX Research Inc

[48] Warmer CJ, Hommelberg MPF, Roossien B et al (2007) A field test using agents for coordination of residential micro-chp. In: Proceedings of the 2007 international conference on intelligent systems applications to power systems (ISAP'07), Niigata, 5-8 Nov 2007, 4 pp

[49] Roossien B (2009) Field-test upscaling of multi-agent coordination in the electricity grid. In: Proceedings of the 20th international conference and exhibition on electricity distribution (CIRED'09), Part 1, Prague, 8-11 June 2009, 4 pp

[50] Kok K (2013) The PowerMatcher: smart coordination for the smart electricity grid. Ph.D. Thesis. Amsterdam Vrije Universiteit, Amsterdam

[51] Van Pruissen O, Kok K, Eisma A (2015) Simultaneous imbalance reduction and peak shaving using a field operational virtual power plant with heat pumps. In: Proceedings of the $23 \mathrm{rd}$ international conference on electricity distribution, Lyon, 15-18 June 2005, 5 pp

[52] Pentland A (2015) Simple market models fail the test. Nature 525(7568):190-191

[53] Krause SM, Börries S, Bornholdt S (2013) Econophysics of adaptive power markets: when a market does not dampen fluctuations but amplifies them. Phys Rev E 92(1):5

[54] Faruqui A, Hledik RM, Levy A et al (2011) Will smart prices induce smart charging of electric vehicles? Social Science Research Network (SSRN), 25 Aug 2011

[55] Yu RS, Yang WX, Rahardja S (2012) A statistical demand-price model with its application in optimal real-time price. IEEE Trans Smart Grid 3(4):1734-1742

[56] Universal Smart Energy Framework (USEF). http://www.usef. info

[57] Open ADR. http://www.openadr.org/

[58] Energy flexibility platform \& interface. PowerMatching Suite. http://flexiblepower.github.io/EF-Pi\%20Whitepaper/

[59] Chao HP (2011) Demand response in wholesale electricity markets: the choice of customer baseline. J Regul Econ 39(1):68-88

[60] The demand response baseline (2009) White Paper. EnerNOC Inc, Boston
Junjie HU received the M.Sc. degree in Control Theory and Control Engineering from TongJi University, China in 2010 and the Ph.D degree in Electrical Engineering from Technical University of Denmark, Denmark in 2014. Currently, he is a Postdoctoral researcher with the Department of Electrical Engineering, Technical University of Denmark. His main fields of interests are distributed energy resources (DERs) integration with focus on electric vehicles, application of optimal control theory on active distribution grid operation and management, and DER's optimal participation into the ancillary service market.

Guangya YANG received the B.Sc., M.Sc. and Ph.D in 2002, 2005 and 2008 respectively, all in electric power system field. Currently, he is associate professor with the Center for Electric Power and Energy, Department of Electrical Engineering of the Technical University of Denmark. His fields of interest include power system operation and control, renewable energy integration and wide-area system monitoring and protection.

Koen KOK is a scientific researcher in the interdisciplinary field of intelligent electricity systems, combining electrical engineering and control engineering with computational intelligence. Currently, he is a Senior Scientist at the Center for Electric Power and Energy, Technical University of Denmark. Further, he is affiliated with TNO, the largest applied research institute in The Netherlands, where he is a senior scientist as well. He holds a B.Sc. in Electrical Engineering and an M.Sc. and a Ph.D in Computer Science. He has extensive research experience in the fields of market-based control of power systems, smart grid ICT architectures and integration of distributed energy resources and demand response in the electricity system. He is one of the inventors of the PowerMatcher, an award winning and opensource smart grid technology for matching demand and distributed generation on one hand with available renewable power and network capacity on the other.

Yusheng XUE received the M.Sc. degree in Electrical Engineering from EPRI, China in 1981 and the Ph.D degree from the University of Liege, Belgium in 1987. He has been an Academician of the Chinese Academy of Engineering since 1995. He is now the Honorary President of State Grid Electric Power Research Institute (SGEPRI or NARI), China, Adjunct Professor in dozens of Chinese universities and an Adjunct Professor of the University of Newcastle in Australia. $\mathrm{He}$ is the Editor-in-Chief of Automation of Electric Power System (in Chinese) and Journal of Modern Power Systems and Clean Energy (in English), as well as Chairman of the Technical Committee of Chinese National Committee of CIGRE since 2005.

Henrik W. BINDNER received the M.S. degree in electrical engineering from the Technical University of Denmark, Lyngby, Denmark, in 1988. Since 1990, he has been with the Risø National Laboratory for Sustainable Energy, Roskilde, Denmark, in the Wind Energy Division. Since 2008, he has been a Senior Scientist with the Department for Electrical Engineering at the Ris $\varnothing$ Campus, Technical University of Denmark. He has mainly been working on integration of wind energy into power systems, as well as analysis, design, and control of small island systems. 